\title{
The efficiency and safety of immune checkpoint inhibitors in the treatment of small cell lung cancer: a meta-analysis
}

\author{
Shanshan Zhang, Minghong Bi \\ Department of Oncology, the First Affiliated Hospital of Bengbu Medical College, Bengbu, China \\ Contributions: (I) Conception and design: S Zhang; (II) Administrative support: M Bi; (III) Provision of study materials or patients: S Zhang; (IV) \\ Collection and assembly of data: S Zhang; (V) Data analysis and interpretation: S Zhang; (VI) Manuscript writing: All authors; (VII) Final approval \\ of manuscript: All authors. \\ Correspondence to: Minghong Bi. Department of Oncology, the First Affiliated Hospital of Bengbu Medical College, Bengbu, China. \\ Email: bmh2003@126.com.
}

\begin{abstract}
Background Small cell lung cancer (SCLC) is highly invasive and fatal, sensitive to chemotherapy and radiotherapy but prone to relapse, with a poor overall survival rate. It is particularly urgent for SCLC patients to receive effective follow-up treatment. In the past 20 years, there has been no breakthrough in clinical treatment of SCLC. Currently, clinical studies on immunotherapy for SCLC with extensive stage disease (ED) have achieved good efficacy, bringing new hope for the treatment of small-cell lung cancer. PD-1 inhibitors used to treat small cell lung cancer include Pembrolizumab and Nivolumab. PD-L1 inhibitors mainly include Atezolizumab and Durvalumab. Other PD-1/PD-L1 inhibitors, such as Avelumab, are currently being tried for SCLC and the results have not yet been published. This study is to evaluate the efficacy and safety of immunotherapy in patients with ED SCLC.
\end{abstract}

Methods: A literature search of the PubMed, Embase, and Cochrane Library databases were performed. Two reviewers independently screened the literature, extracted the data, and evaluated the risk of bias of the included studies. RevMan 5.3 software was used for meta-analysis.

Results: Four studies involving 1,981 patients with ED SCLC were included. Both overall survival (OS) [hazard ratio $(\mathrm{HR})=0.80,95 \%$ confidence interval $(\mathrm{CI})(0.68,0.95), \mathrm{P}=0.009$ ] and progression-free survival (PFS) $[\mathrm{HR}=0.82,95 \% \mathrm{CI}(0.75,0.90), \mathrm{P}<0.00001]$ were longer in the immunotherapy group than in the chemotherapy group. The incidence of total treatment-related adverse events in the immunotherapy group were lower than those in the chemotherapy group [relative risk $(\mathrm{RR})=1.050,95 \% \mathrm{CI}(1.010,1.080)$, $\mathrm{P}=0.007]$, and the differences were statistically significant.

Conclusions: Immunotherapy has better efficacy and safety than chemotherapy for the treatment of ED SCLC.

Keywords: Immunotherapy; chemotherapy; small cell lung cancer

Submitted Sep 14, 2020. Accepted for publication Nov 06, 2020.

doi: 10.21037/apm-20-2011

View this article at: http://dx.doi.org/10.21037/apm-20-2011

\section{Introduction}

In recent years, the incidence and mortality rates of lung cancer have been increasing. Small cell lung cancer (SCLC) accounts for about $15 \%$ of all lung cancers, and is highly invasive and fatal due to its rapid growth, abundant stem cells, and gene instability (1). Moreover, at the time of diagnosis, most of the patients are in the extensive stage (when tumor cells have spread beyond the supraclavicular region), and for these patients, the 5 -year survival rate is less than $2 \%(2,3)$. Due to the slow progress of research and the entry stage of treatment, effective follow-up treatment for SCLC patients is particularly urgent. Etoposide plus cisplatin (EP) chemotherapy is the standard treatment for extensive stage disease (ED) SCLC. Although the response 
rate is high, most of the cases recur within one year. Chemotherapy drugs such as irinotecan, cyclophosphamide, adriamycin, and vincristine (CAV) have been used as secondline therapy, but have only produced minor effects (4). With increased research into surgery, radiotherapy, chemotherapy, and targeted therapy, the emergence of immunotherapy may become a new pillar of cancer treatment (5). In some international randomized controlled trials (RCTs), immunotherapy has been shown to be a safe and effective strategy to restore the antineoplastic activity of T lymphocytes at key immune checkpoints, and can be used in patients with advanced or metastatic malignancies (6-10).

At present, immune checkpoint inhibitors stand out from many anti-tumor research, and have made breakthrough progress, and change the clinical practice, with epochmaking significance. The research on SCLC immune checkpoint inhibitors mainly focuses on CTLA-4 inhibitors and PD-1/PD-L1 inhibitors (11). As a negative regulator of $\mathrm{T}$ cell activation expressed on the surface of $\mathrm{T}$ cells, the cytotoxic T-lymphocyte-associated 4 (CTLA-4) protein competitively inhibits $\mathrm{T}$ cell activation with cluster of differentiation 28 (CD28). The combination of CTLA-4 inhibitors and CTLA-4 blocks the interaction between the latter and its ligand (CD80/CD86), which could block the negative regulatory signal of CTLA-4, and thereby enhance the antineoplastic activity of $\mathrm{T}$ cells (12). The interaction after the binding of programmed cell death protein 1 (PD-1) and its ligand (PD-L1 or PD-L2) in the host tissue inhibits $T$ cell receptor (TCR) signals. This leads to functional impairment of the effectors in CD4+ and CD8+ T cells, limits the interaction between $\mathrm{T}$ cells and target cells, and inactivates $T$ cells and inhibits their proliferative ability (13-15). PD-1/PD-L1 inhibitors can block this negative regulatory signal, activate CD8 $+\mathrm{T}$ cells (TEX) with low function, and restore their anti-tumor function, thereby enhancing the immune response (16).

Keynote-028 study showed that pembrolizumab had good anticancer activity in PD-L1 positive patients with extensive SCLC, and the objective remission rate could reach $33 \%$. The national comprehensive cancer network (NCCN) guidelines recommend ipilimumab and nivolumab for refractory patients (category 2a) who have been treated for less than 6 months from treatment to recurrence (17). An open, randomized, phase III study of nivolumab controlled chemotherapy for relapsed SCLC after firstline platinum containing chemotherapy regimen is under way in the ongoing phase III clinical trial, and the phase III study of PD-L1 inhibitor atezolizumab + carboplatin
+ etoposide in the treatment of extensive stage SCLC is under way. These results will establish the status of immunotherapy in the combination therapy of ES-SCLC, and search for biomarkers that can predict the benefits from immunotherapy, which will promote the development of this field.

In the aspect of small cell lung cancer, there are few studies on the single immunotherapy, combined chemotherapy and chemotherapy alone, and there are more disputes about the effectiveness and safety of combined treatment, which needs meta-analysis and statistical analysis. This study aims to compare several international large-scale randomized controlled trials (RCTs) related to immunotherapy combined with chemotherapy, and analyze whether the effectiveness and safety are statistically significant, so as to provide treatment reference for readers. We present the following article in accordance with the PRISMA reporting checklist (available at http://dx.doi. org/10.21037/apm-20-2011).

\section{Methods}

\section{Data and metbods}

\section{Inclusion criteria}

(I) Studies involving patients diagnosed as ED SCLC by pathology and cytology; (II) clinical trials published comparing ICIs and chemotherapy for the treatment of ED SCLC; (III) randomized controlled trials (RCTs) in phase II or III; (IV) studies exploring more than one main outcome index: overall survival (OS), progression-free survival (PFS), or objective response rate (ORR).

\section{Exclusion criteria}

(I) Studies involving patients that received anti-tumor immunotherapy before joining the clinical trial; (II) Studies involving treatment measures such as combined irradiation, molecular targeted therapy, or other ICI treatment; (III) Republished clinical research; (IV) Research with incomplete data or where we were unable to extract the relevant data.

\section{Retrieval strategies}

We performed a comprehensive literature search on the PubMed, Embase, and Cochrane Library databases, covering all articles published on these databases up to March $1^{\text {st }}, 2020$. Our key word search terms involved a combination of subject and free words, including Small Cell Lung Cancer, Oat Cell Lung Cancer, Small Cell Cancer 
Table 1 Quality assessment of the included randomized controlled trials (RCTs)

\begin{tabular}{lcccccc}
\hline Study & $\begin{array}{c}\text { Randomized } \\
\text { method }\end{array}$ & Blinding & $\begin{array}{c}\text { Random sequence } \\
\text { generation }\end{array}$ & $\begin{array}{c}\text { Allocation } \\
\text { concealment }\end{array}$ & $\begin{array}{c}\text { Incomplete outcome Free of selective } \\
\text { data addressed }\end{array}$ & $\begin{array}{c}\text { Free of } \\
\text { other bias }\end{array}$ \\
\hline Horn et al. (18); [2018] & Yes & No & Yes & Unclear & No & Unclear \\
Paz-Ares et al. (19); [2019] & Yes & No & Yes & Unclear & No & No Unclear \\
Reck et al. (20); [2013] & Yes & No & Yes & Unclear & No & No \\
Reck et al. (21); [2016] & Yes & No & Yes & Unclear & No & No Unclear \\
\hline
\end{tabular}

of The Lung, Carcinoma, Small Cell Lung, Anti CTLA-4 Mab Ipilimumab, MDX-CTLA-4, pembrolizumab, SCH-900475, Keytruda, MK-3475, Lambrolizumab, Atezolizumab, anti-PDL1, MPDL3280A, Tecentriq, RG7446, Durvalumab, MEDI4736, Imfinzi, Nivolumab, Opdivo, ONO-4538, MDX-1106, BMS936558, CD279 Antigen, PD1 Receptor, Programmed Cell Death 1 Protein, B7 H1 Antigen, CD274 Antigen, B7H1 Immune Costimulatory Protein, etc.

\section{Quality evaluation of included literature}

Two reviewers independently screened the literature and evaluated the quality and bias of the included studies according to the standards recommended by the Cochrane manual. The main evaluation methods are shown in Table 1 .

\section{Statistical methods}

RevMan 5.3 software was used to statistically analyze the data. Hazard ratios (HR) or relative risks (RR) were used as the effect indexes for comparison between the immunotherapy and chemotherapy groups. Point estimates and $95 \%$ confidence intervals (CI) of each effect size were given. We tested the statistical heterogeneity of the included studies using the $\mathrm{I}^{2}$ statistic. Studies were considered to show no statistical heterogeneity if $\mathrm{P}>0.10$ and $\mathrm{I}^{2}<50 \%$, and a fixed effects model was used for combined analysis. However, $\mathrm{P} \leq 0.10$ or $\mathrm{I}^{2} \geq 50 \%$ indicated a statistical heterogeneity among the studies, and a random effects model was employed. Significant clinical heterogeneity was examined by subgroup, sensitivity, or descriptive analysis. Differences were statistically significant at $\mathrm{P}<0.05$.

\section{Results}

\section{Literature screening results}

A total of 2,282 articles were initially screened. These articles were examined further by reading the titles and abstracts. Finally, four clinical RCTs in phase II or III were included in the meta-analysis (Figure 1).

\section{Basic information of the included studies}

A total of four RCTs involving 1,981 patients with ED SCLC were selected for our meta-analysis, including 989 cases in the immunotherapy group and 992 cases in the chemotherapy group. The basic information of the included studies are shown in Table 2. Briefly, Horn et al. (18) is a RCT comparing atezolizumab and chemotherapy; Paz-Ares et al. (19) included two subgroups: durvalumab + tremelimumab + platinum/etoposide and durvalumab + platinum/etoposide. From this paper, only a comparison between the durvalumab group and chemotherapy group was included; Reck et al. (20) is a RCT comparing ipilimumab and chemotherapy. In this paper, only the staged groups were included in the analysis (although the original text included two simultaneous and staged subgroups); Martin Reck et al. (21) is a phase III RCT comparing ipilimumab and chemotherapy.

\section{Quality of the included studies}

The sample sizes of the four RCT studies were sufficient, and the randomized controlled method was adopted. All four studies were open trials and were performed in accordance with the principle of random distribution. The data were complete without selectively reporting results. However, it was unclear whether there was distribution hiding or other biases.

\section{Meta-analysis results}

Overall survival (OS)

All four RCTs reported OS. Meta-analysis using a random 


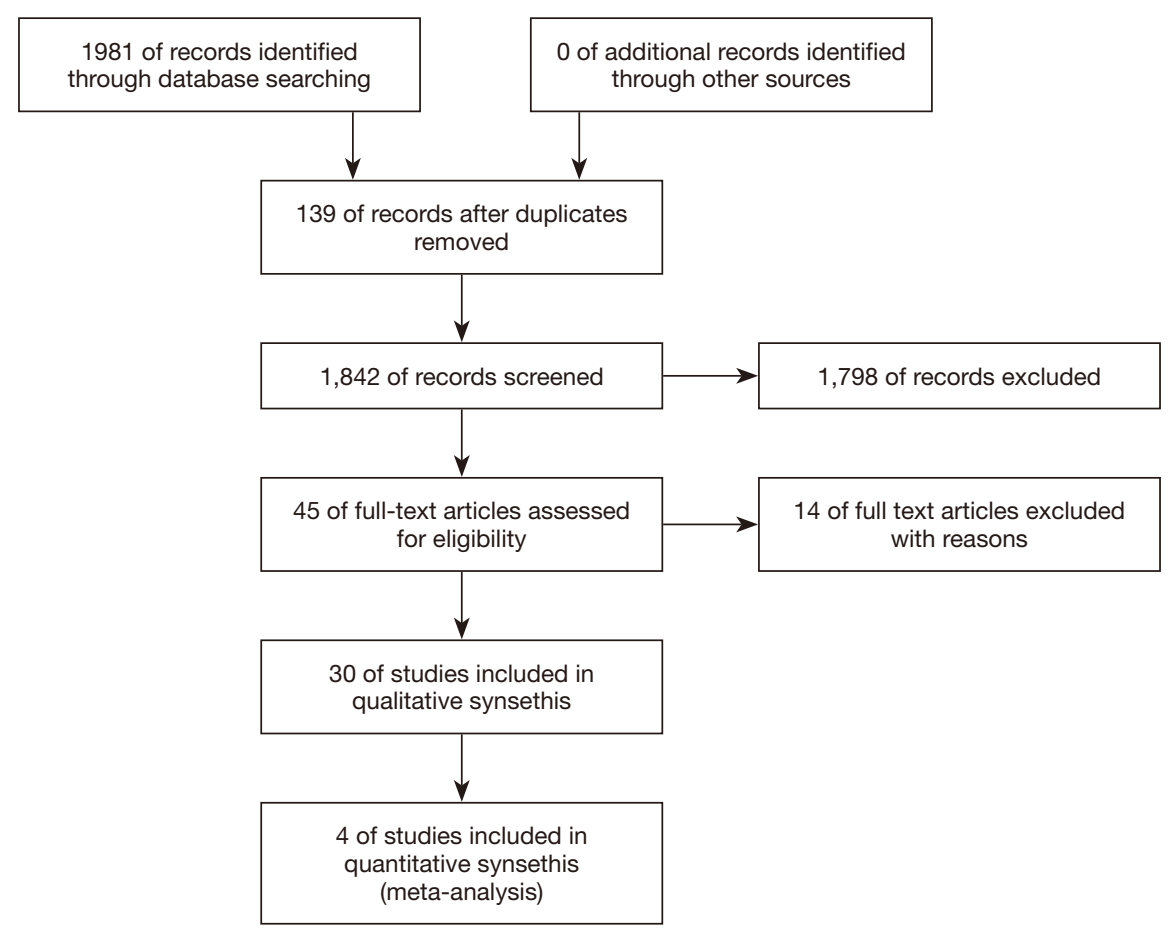

Figure 1 Flow chart of the search strategy and study selection.

Table 2 Basic information of the included studies

\begin{tabular}{|c|c|c|c|c|c|c|}
\hline \multirow{2}{*}{ Study } & \multirow{2}{*}{ Design } & \multirow{2}{*}{ Phase } & \multicolumn{2}{|c|}{ Number of patients (n) } & \multicolumn{2}{|c|}{ Therapeutic regimen } \\
\hline & & & Immunotherapy & Chemotherapy & Immunotherapy & Chemotherapy \\
\hline Horn et al. (18); [2018] & RCT & III & 201 & 202 & Atezo: $1,200 \mathrm{mg}$, day 1 & $\begin{array}{c}\text { Carbo: day } 1 \text {. Eto: } 100 \mathrm{mg} / \mathrm{m}^{2} \text {, } \\
\text { days } 1-3\end{array}$ \\
\hline Paz-Ares et al. (19); [2019] & $\mathrm{RCT}$ & III & 268 & 269 & Dur: $1,500 \mathrm{mg}$, Tiw & $\begin{array}{c}\text { Carbo: day } 1 \text {. Eto: } 80-100 \mathrm{mg} / \mathrm{m}^{2} \text {, } \\
\text { days } 1-3\end{array}$ \\
\hline Reck et al. (21); [2016] & $\mathrm{RCT}$ & III & 478 & 476 & Ipi: 10mg/kg, Tiw & $\begin{array}{c}\text { PT-C: day 1. Eto: } 100 \mathrm{mg} / \mathrm{m}^{2} \text {, } \\
\text { days } 1-3\end{array}$ \\
\hline
\end{tabular}

RCT, randomized controlled trial; Ipi, Ipilimumab; Atezo, Atezolizumab; Dur, Durvalumab; Carbo, Carboplatin; Eto, Etoposide; Pacl, Paclitaxel; Day 1, day 1 of each cycle; Day 1-3, days 1 to 3 of each cycle; PT-C, Platinum-based chemotherapy; Tiw, every three weeks.

effects model showed that the OS of the immunotherapy group was higher than that of the first-line chemotherapy group in the treatment of SCLC, and the difference was statistically significant $[\mathrm{HR}=0.80,95 \% \mathrm{CI}(0.68,0.95)$, $\mathrm{P}=0.009$, Figure 2].

\section{PFS}

All four RCTs reported PFS. Meta-analysis using a random effects model showed that the PFS of the immunotherapy group was higher than that of the first-line chemotherapy group in the treatment of SCLC, and the difference was statistically significant $[\mathrm{HR}=0.82,95 \%$ CI $(0.75,0.90)$, $\mathrm{P}<0.00001$, Figure 3].

\section{ORR}

All four RCTs reported ORR. Meta-analysis using a random effects model showed that there was no significant difference between the immunotherapy group and the first-line chemotherapy group in the treatment of SCLC $[\mathrm{RR}=1.04,95 \% \mathrm{CI}(0.93,1.17), \mathrm{P}=0.46$, Figure 4]. 


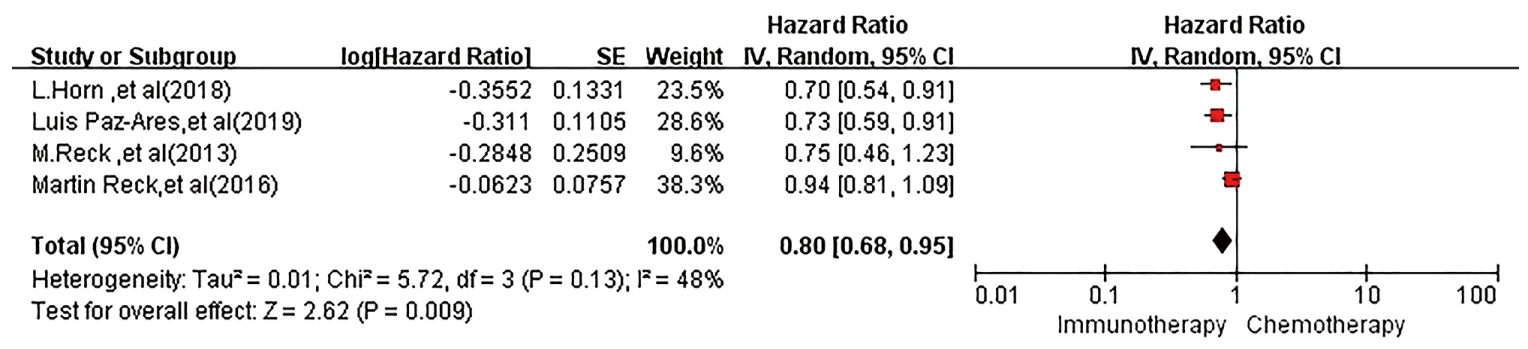

Figure 2 Forest plot of overall survival (OS) in patients with ED SCLC after immunotherapy vs. chemotherapy. SE, standard error; HR, hazard ratio; CI, confidence interval; ED, extensive stage disease; SCLC, small cell lung cancer.

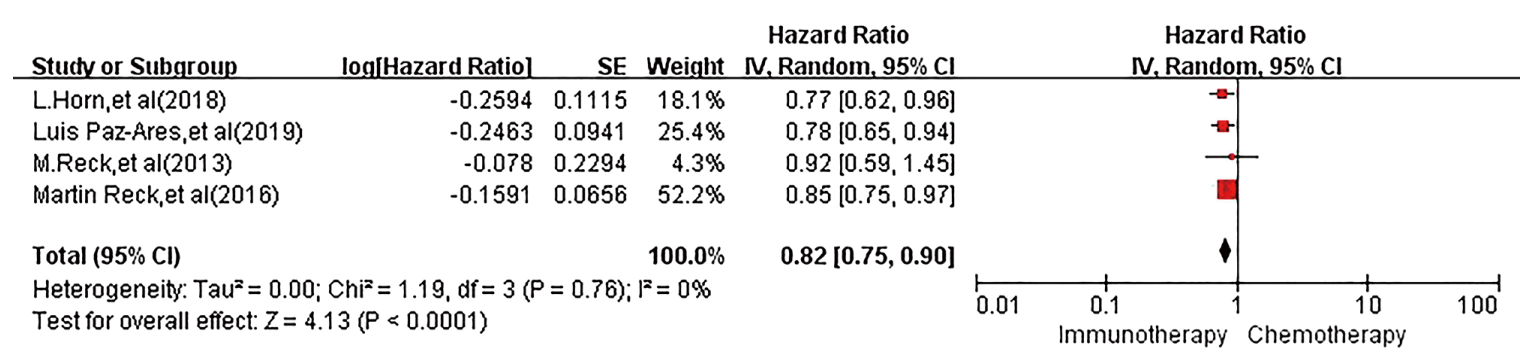

Figure 3 Forest plot of progression-free survival (PFS) in patients with ED SCLC after immunotherapy vs. chemotherapy. SE, standard error; HR, hazard ratio; CI, confidence interval; ED, extensive stage disease; SCLC, small cell lung cancer.

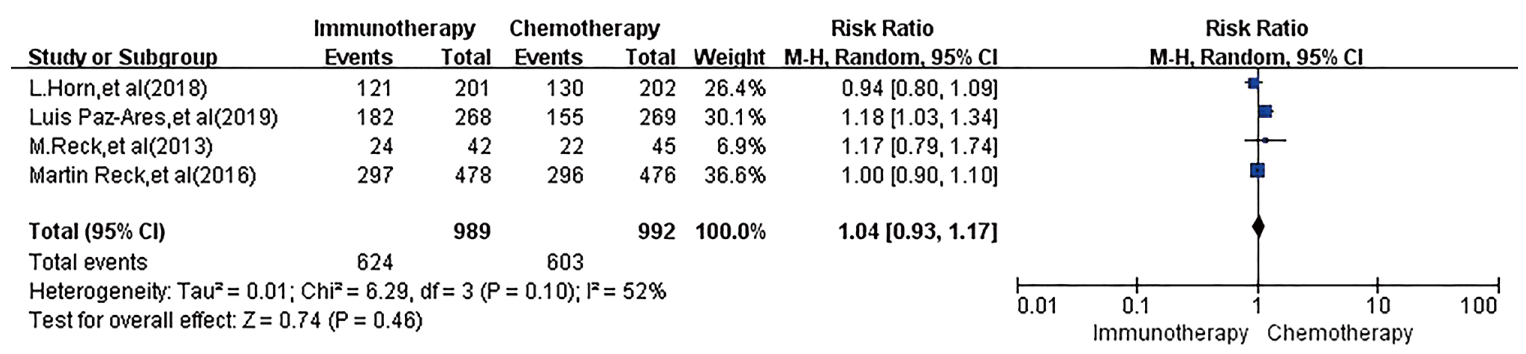

Figure 4 Forest plot of objective response rate (ORR) in patients with ED SCLC after immunotherapy vs. chemotherapy. RR, relative risk; CI, confidence interval; ED, extensive stage disease; SCLC, small cell lung cancer.

\section{Adverse reactions}

All four RCTs reported treatment-related adverse reactions. Meta-analysis using a random effects model showed that the adverse reactions of the immunotherapy group were lower than those of the chemotherapy group, and the difference was statistically significant $[\mathrm{RR}=1.050,95 \% \mathrm{CI}(1.010$, 1.080), $\mathrm{P}=0.007$, Figure 5]. A subgroup analysis of common adverse reactions was conducted, as shown in Table 3. There were no statistically significant differences except for decreased appetite and decreased white-cell count.

\section{Discussion}

Numerous studies have shown that immunosuppression induced by lung cancer cells is a crucial factor in the malignant progression. Immunotherapy can enhance the innate immune response of patients, and is expected to induce a long-term response (22). Chemotherapy has long been considered to be immunosuppressive and incompatible with immunotherapy. In order to improve the low response rate of immune inhibitors (when used alone), some experiments have combined chemotherapy with ICIs, 


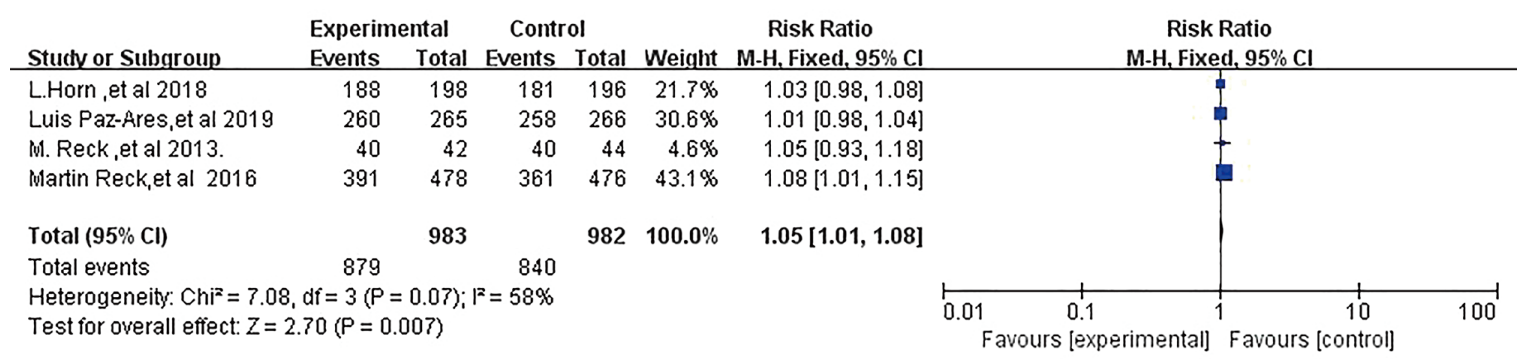

Figure 5 Forest plot of total treatment-related adverse events in patients with ED SCLC after immunotherapy vs. chemotherapy. RR, relative risk; CI, confidence interval; ED, extensive stage disease; SCLC, small cell lung cancer.

Table 3 Meta-analysis of various treatment-related adverse events in patients with ED SCLC after immunotherapy vs. chemotherapy

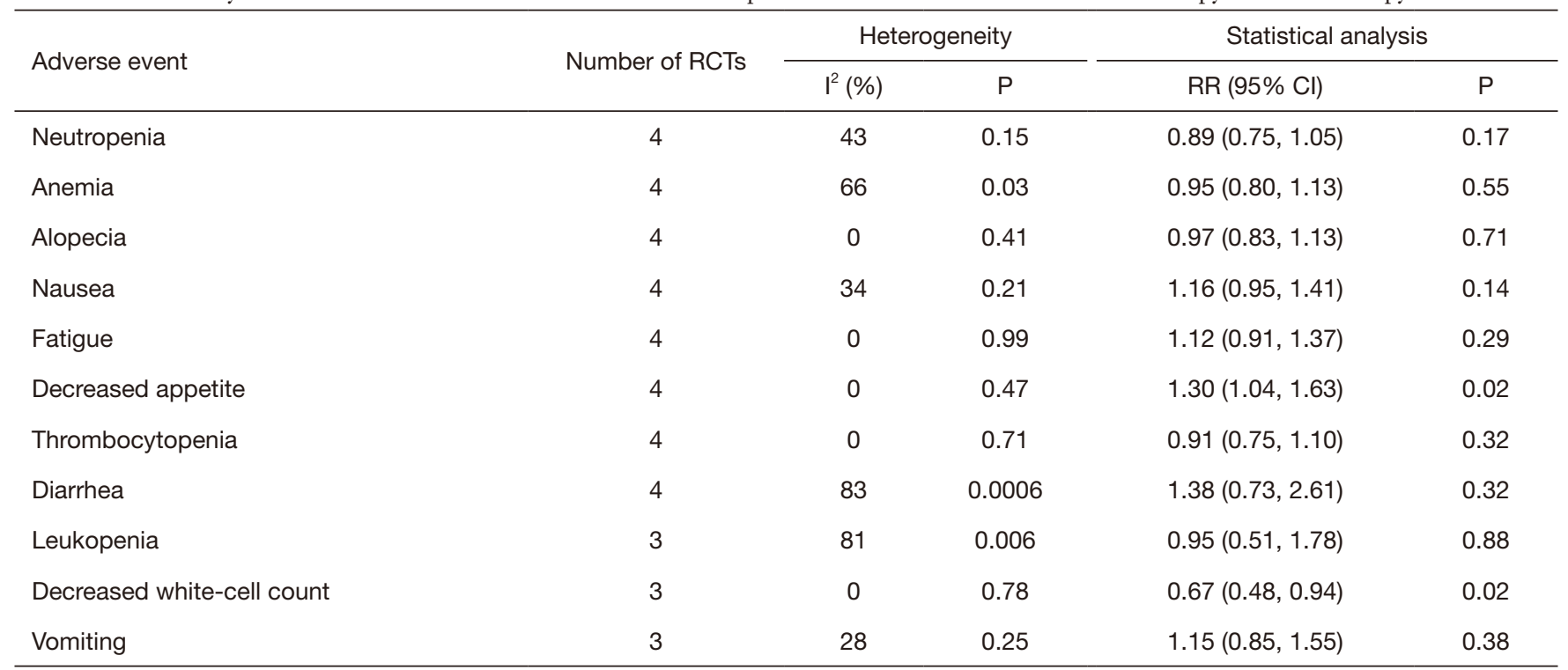

ED, extensive stage disease; SCLC, small cell lung cancer; RCT, randomized controlled trial.

attempting to utilize the immunomodulatory (synergistic) effect of chemotherapy on tumor and immune cells (23).

In recent years, there have been an increasing number of clinical trials regarding immunotherapy of SCLC. PD-1 inhibitors (nivolumab and pembrolizumab) and PD-L1 inhibitors (durvalumab and atezolizumab) have shown antineoplastic activity and manageable safety in various tumor types (24). The clinical trials currently being conducted, including Keynote-604, Checkmate 331, and NCT02963090, will provide more evidence regarding the treatment of SCLC (5).

In the four clinical trials included in our metaanalysis, both OS and PFS improved, thus reinforcing the combination therapy as a potentially novel strategy for the treatment of SCLC. Among them, the efficacy for ipilimumab + paclitaxel + carboplatin in Reck et al. was significantly improved. However, the OS rate of ipilimumab + chemotherapy was not significantly different from that of chemotherapy alone. This is understandable, considering that ipilimumab targets CTLA-4 to stimulate the activation of peripheral blood $\mathrm{T}$ cells, but cannot activate $\mathrm{T}$ cells in the tumor microenvironment. In view of this mechanism of action, Reck et al. speculated that the value of ipilimumab on chemotherapy synergistic effect was limited (21). Subsequent studies have shown that nivolumab and ipilimumab may provide a synergistic antitumor effect (25). Combination chemotherapy with ipilimumab, a CTLA4 inhibitor, did not show obvious synergistic effect, while the PD-1 inhibitor for tumor-infiltrating lymphocytes supplemented the antineoplastic activity of CTLA- 
4 inhibitor in a non-redundant way; the related clinical research (Checkmate 451) is currently being conducted (26). The results of IMpower133 (18) and CASPIAN (19) have shown that platinum and etoposide might not deplete $\mathrm{T}$ cells in the tumor, and atezolizumab and durvalumab could activate $T$ cells in the tumor and play an anti-tumor role, however this requires further research.

According to the results of our meta-analysis, immunotherapy performed better than chemotherapy in OS $\mathrm{HR}=0.800,95 \% \mathrm{CI}(0.680,0.950), \mathrm{P}=0.009]$ and PFS [HR $=0.820,95 \%$ CI $(0.750,0.900), \mathrm{P}<0.001]$. Furthermore, the incidence of adverse reactions in the immunotherapy group was better than the chemotherapy group $[R R=1.050$, $95 \%$ CI $(1.010,1.080), \mathrm{P}=0.007]$. This suggests that combining checkpoint inhibition with cytotoxic therapy in induction may be beneficial, with outcomes consistent with international trials. Atezolizumab was launched in China in 2020, and Atezolizumab combined with chemotherapy became the preferred National Comprehensive Cancer Network (NCCN) first-line treatment of ED SCLC (16), providing stronger support for the clinical application of immunotherapy combined with chemotherapy.

The four studies included in this paper were large RCTs with rigorous study designs. However, due to limited immunotherapy studies on SCLC and ongoing partial trials, there were no clear outcome indicators, and thus the number of included studies was relatively small, resulting in bias. Therefore, the efficacy and safety of immunological preparations should be comprehensively assessed and carefully selected.

\section{Acknowledgments}

Funding: Anhui University Natural Science Research Project (KJ2019A0372).

\section{Footnote}

Reporting Checklist: The authors have completed the PRISMA reporting checklist. Available at http://dx.doi. org/10.21037/apm-20-2011

Conflicts of Interest: Both authors have completed the ICMJE uniform disclosure form (available at http://dx.doi. org/10.21037/apm-20-2011). The authors have no conflicts of interest to declare.

Ethical Statement: The authors are accountable for all aspects of the work in ensuring that questions related to the accuracy or integrity of any part of the work are appropriately investigated and resolved.

Open Access Statement: This is an Open Access article distributed in accordance with the Creative Commons Attribution-NonCommercial-NoDerivs 4.0 International License (CC BY-NC-ND 4.0), which permits the noncommercial replication and distribution of the article with the strict proviso that no changes or edits are made and the original work is properly cited (including links to both the formal publication through the relevant DOI and the license). See: https://creativecommons.org/licenses/by-nc-nd/4.0/.

\section{References}

1. Behera M, Ragin C, Kim S, et al. Trends, predictors, and impact of systemic chemotherapy in small cell lung cancer patients between 1985 and 2005. Cancer 2016;122:50-60.

2. Sabari JK, Lok BH, Laird JH, et al. Unravelling the biology of SCLC: implications for therapy. Nat Rev Clin Oncol 2017;14:549-61.

3. Farago AF, Keane FK. Current standards for clinical management of small cell lung cancer. Transl Lung Cancer Res 2018;7:69-79.

4. Wang Q, Peng W, Jiang M, et al. Research progress of immunotherapy and prognostic markers in small cell lung cancer. Zhongguo Fei Ai Za Zhi 2020;23:182-8.

5. Regzedmaa O, Zhang $\mathrm{H}$, Liu $\mathrm{H}$, et al. Immune checkpoint inhibitors for small cell lung cancer: opportunities and challenges. Onco Targets Ther 2019;12:4605-20.

6. Fang W, Zhao S, Zhang Y, et al. Ongoing Phase I Studies of Immune Checkpoint Inhibitors in China. Oncologist 2019;24:S11-S20.

7. El-Khoueiry AB, Sangro B, Yau T, et al. Nivolumab in patients with advanced hepatocellular carcinoma (CheckMate 040): an open-label, non-comparative, phase 1/2 dose escalation and expansion trial. Lancet 2017;389:2492-502.

8. Ferris RL, Blumenschein G Jr, Fayette J, et al. Nivolumab for recurrent squamous-cell carcinoma of the head and neck. N Engl J Med 2016;375:1856-67.

9. Sharma P, Callahan MK, Bono P, et al. Nivolumab monotherapy in recurrent metastatic urothelial carcinoma (CheckMate 032): a multicentre, open-label, two-stage, multi-arm, phase 1/2 trial. Lancet Oncol 2016;17:1590-8.

10. Rosenberg JE, Hoffman-Censits J, Powles T, et al. Atezolizumab in patients with locally advanced and 
metastatic urothelial carcinoma who have progressed following treatment with platinum-based chemotherapy: a single-arm, multicentre, phase 2 trial. Lancet 2016;387:1909-20.

11. Reck M, Heigener D, Reinmuth N. Immunotherapy for small-cell lung cancer: emerging evidence. Future Oncol 2016;12:931-43.

12. Hellmann MD, Ciuleanu TE, Pluzanski A, et al. Nivolumab plus ipilimumab in lung cancer with a high tumor mutational burden. N Engl J Med 2018;378:2093-104.

13. Kamphorst AO, Wieland A, Nasti T, et al. Rescue of exhausted CD8 T cells by PD-1-targeted therapies is CD28-dependent. Science 2017;355:1423-7.

14. Hui E, Cheung J, Zhu J, et al. T cell costimulatory receptor CD28 is a primary target for PD-1-mediated inhibition. Science 2017;355:1428-33.

15. Ribas A. Tumor immunotherapy directed at PD-1. N Engl J Med 2012;366:2517-2519.

16. Wherry EJ, Kurachi M. Molecular and cellular insights into T cell exhaustion. Nat Rev Immunol 2015;15:486-99.

17. Ott PA, Elez E, Hiret S, et al. Pembrolizumab in patients with extensive-stage small-cell lung cancer:results from the phase Ib KEYNOTE-028 Study. J Clin Oncol 2017;35:3823-9.

18. Horn L, Mansfield AS, Szczęsna A, et al. First-line atezolizumab plus chemotherapy in extensive-stage smallcell lung cancer. N Engl J Med 2018;379:2220-9.

19. Paz-Ares L, Dvorkin M, Chen Y, et al. Durvalumab plus platinum-etoposide versus platinum-etoposide in firstline treatment of extensive-stage small-cell lung cancer (CASPIAN): a randomised, controlled, open-label, phase 3 trial. Lancet 2019;394:1929-39.

20. Reck M, Bondarenko I, Luft A, et al. Ipilimumab in combination with paclitaxel and carboplatin as first-line therapy in extensive-disease-small-cell lung cancer: results from a randomized, double-blind, multicenter phase 2 trial. Ann Oncol 2013;24:75-83.

21. Reck M, Luft A, Szczesna A, et al. Phase III randomized trial of ipilimumab plus etoposide and platinum versus placebo plus etoposide and platinum in extensive-stage small-cell lung cancer. J Clin Oncol 2016;34:3740-8.

22. Li Y, Wu YL. Immunotherapy for small-cell lung cancer. Lancet Oncol 2016;17:846-7.

23. Selby MJ, Engelhardt JJ, Quigley M, et al. Anti-CTLA-4 antibodies of IgG2a isotype enhance antitumor activity through reduction of intratumoral regulatory $\mathrm{T}$ cells. Cancer Immunol Res 2013;1:32-42.

24. Goldberg S. IBS09. 02 Immunotherapy for small cell and neuroendocrine tumors. J Thorac Oncol 2019;14:S99.

25. Rizvi NA, Gettinger SN, Goldman J W, et al. Safety and efficacy of first-line nivolumab (NIVO; anti-programmed death-1 (PD-1)) and ipilimumab in non-small cell lung cancer (NSCLC). J Thorac Oncol 2015;1:S176.

26. Ready N, Owonikoko TK, Postmus PE, et al. CheckMate 451: A randomized, double-blind, phase III trial of nivolumab (nivo), nivo plus ipilimumab (ipi), or placebo as maintenance therapy in patients (pts) with extensivestage disease small cell lung cancer (ED-SCLC) after firstline platinum-based doublet chemotherapy (PT-DC). Clin Oncol 2016;34:abstr TPS5879.

(English Language Editor: A. Kassem)
Cite this article as: Zhang S, Bi M. The efficiency and safety of immune checkpoint inhibitors in the treatment of small cell lung cancer: a meta-analysis. Ann Palliat Med 2020;9(6):4081-4088. doi: 10.21037/apm-20-2011 